www. revis tad y o. com

\title{
Universidad corporativa y aprendizaje organizacional: un marco de refe- rencia
}

\author{
Corporate university and organizational learning: a theoretical framework
}

Carmenza Luna-Amaya ${ }^{1}$, Ricardo de la Hoz¹, Angello Gómez-Velásquez ${ }^{1}$, Andrés Manjarrés-Salas , Julio Vidal' ${ }^{1}$, Olga Jaramillo² y Carmen Berdugo-Correa ${ }^{1}$

${ }^{1}$ Departamento de Ingeniería Industrial. Universidad del Norte, Barranquilla, Colombia.

${ }^{2}$ Departamento de Negocios. Universidad del Norte, Barranquilla, Colombia

cluna@uninorte.edu.co, rdelahoz@unilibrebaq.edu.co, r9angello@hotmail.com, aemanjarres89@hotmail.com, julio.vidal.pombo@hotmail. com, oljarami@uninorte.edu.co, cberdugo@uninorte.edu.co

Fecha de recepción: 09-12-2014

Fecha de aceptación: 22-02-2016

Resumen: El talento humano juega un papel fundamental dentro de la estructura operativa, táctica y estratégica de las organizaciones, las cuales están apuntando a contar con personal mejor cualificado y capaz de hallar soluciones a los problemas y retos que enfrenta para el sostenimiento de su ventaja competitiva. En este contexto, el aprendizaje organizacional y la universidad corporativa, como herramientas, tienen una tendencia de aplicación cada vez mayor. El presente trabajo realiza un estado del arte sobre dichos conceptos, mostrando sus tendencias de acuerdo a una escala de clasificación definida

Palabras clave: Universidad corporativa, aprendizaje organizacional, entornos virtuales de aprendizaje, competencias

Abstract: Human talent plays a key role in the operational, tactical and strategic structure of organizations, which are targeting to have a better qualified staff who find solutions to problems and face challenges to sustain its competitive advantage. In this context, Organizational Learning and Corporate University, as tools, have a trend of increasing application. In this vein, this paper aims to give the readers a conceptual framework about the application and state of the art on Corporate Universities and Organizational Learning.

Applying the methodology of classification proposed by Lage and Godinho (2010) in their research, different sources were analyzed and classified in order to develop a theoretical framework. In particular, the papers were classified according to seven topics: a) Geographical location in which the research was developed, which refers to the place where its subject of study was located (America, Europe, Asia, Africa, Middle East and/or Oceania); b) Sector in which the research was conducted, that is, the sector where the Corporate University was created, taking into account sub-levels of classifications (textile, automobile and metal-mechanical industries, healthcare industry and educational sector); c) Main topic of research, which was divided into five (5) categories (Integral organizational learning systems, Virtual learning environments, Corporate university, Enterprise-university programs and Information Technologies (IT's) used); d) Educational strategy, which represents all educational strategies raised in the research related to the topics under study, using sub-categories as Training Programs, Virtual Education programs, Classroom programs in higher education and Hybrid educational development programs; e) Related information technologies, which deals with the use of ICT in the process of educational development and using subcategories used for this topic: Virtual Platforms, Implemented programs on the intranet and Platforms on the cell phone; f) Competency-based approach, considering whether or not the program was made based on competencies and g) Results obtained, which refers to the successfully achievement of the results expected when the research was planned.

The consulted literature was classified under the previous mentioned criteria, in order to show the readers the impact of Corporate University and Organizational Learning. The findings of this study and the theoretical framework are shown at the end of this paper. Researchers and practitioners will find this synthesis useful since it can be considered an important aid to analyze the rationale under these approaches for staff developing in the current context of organizations. 
Finally, the results show that there is extensive research about virtual learning environments, continuing progress in corporate universities and there is an open and growing field toward integrated learning systems in organizations. It was found that Corporate Universities show a major growing trend, and it is expected that they could be compared in size with traditional universities in the long term since they have the advantage of offering specific and applied solutions to those problems organizations face day by day.

Keywords: Corporate University, Organizational Learning, Virtual Learning Environments, Competencies

\section{Introducción}

El aprendizaje organizacional es un proceso clave en el fortalecimiento y desarrollo de las competencias del personal pues es el conocimiento un elemento fundamental para facilitar el mejoramiento continuo en las organizaciones. Sin embargo, deben existir estrategias para su gestión; éste se debe transferir y su aplicación debe reflejarse en los niveles de productividad, considerando las condiciones laborales y sociales de cada trabajador (Vásquez y Arango, 2012).

Los programas de universidades corporativas $y$, en general, de aprendizaje organizacional a partir de alianzas estratégicas entre las instituciones de educación superior, los centros de I + D + i y las empresas son clave para que se pueda generar una gestión adecuada del conocimiento, siendo este tipo de unidades el punto de integración entre los mecanismos formales de enseñanza y las competencias de la organización, dado que en esta última el personal posee la experticia y capacidades necesarias para llevar a cabo las actividades medulares del negocio (Ferreiro et al., 2012).

Los entornos virtuales de aprendizaje son una alternativa fundamental en el apoyo de los programas integrales de aprendizaje organizacional ya que, con su uso, la difusión del conocimiento tiene una cobertura mayor por medio de las tecnologías de la información, las cuales son herramientas utilizadas en todos los ámbitos educativos y están al alcance de cualquier persona en forma de un computador, un teléfono celular, tabletas electrónicas, entre otros dispositivos (Razzaq, Zareen y Ramzan, 2013).

El presente artículo realiza una revisión de la literatura relacionada con el aprendizaje organizacional, suministrando un marco de referencia que abarca este concepto y el de universidades corporativas, así como el de entornos virtuales de aprendizaje basados en Tecnologías de la Información y la Comunicación (TIC's) que facilitan la educación y la gestión del conocimiento en las organizaciones.

Este artículo está organizado de la siguiente manera: en la sección 2 se describe formalmente la meto- dología de investigación utilizada para la recolección y clasificación de la información; en la sección 3 se establece el marco conceptual; se muestra la revisión del marco de referencia y la metodología de clasificación en el apartado 4; se muestra la tabulación de la información en el apartado 5 y, finalmente, en el apartado 6 aparece el marco de referencia. El artículo concluye con las principales observaciones derivadas de la revisión realizada.

\section{Metodología}

El desarrollo del estado del arte acerca del aprendizaje organizacional y las universidades corporativas se realizó tomando como guía la metodología de investigación de Lage y Godinho (2010); en general, se desarrollaron los siguientes pasos:

$\varnothing \rightarrow \quad$ Paso 1: Desarrollo del marco conceptual para la investigación

\section{$\varnothing \rightarrow \quad$ Paso 2: Revisión del estado del arte \\ $\varnothing \rightarrow \quad$ Paso 3: Planteamiento del método de clasifi- cación}

$\varnothing \rightarrow \quad$ Paso 4: Organización y tabulación de la información por categorías de clasificación

\section{$\varnothing \rightarrow \quad$ Paso 5: Presentación del marco referencial \\ $\varnothing \rightarrow \quad$ Paso 6: Análisis y conclusiones}

En el trabajo investigativo, se tuvieron en cuenta alrededor de 50 publicaciones científicas, con 20 años de antigüedad para su aprobación, utilizando publicaciones de todos los continentes.

\section{Marco Conceptual}

De acuerdo a lo definido en la metodología, el punto de partida es la elaboración del marco conceptual bajo el cual se realizó la investigación, con el fin de establecer el criterio de selección de la literatura a ser clasificada 
e incluida en el marco referencial. A continuación se presentan las cuatro definiciones fundamentales empleadas en este artículo: Aprendizaje organizacional, Conocimiento, Entorno virtual de aprendizaje y Universidad corporativa.

Conocimiento: Mezcla fluida de experiencia enmarcada, valores, información contextual y percepción experta que proporciona un marco para evaluar e incorporar nuevas experiencias e información (Davenport y Prusac, 1998).

Aprendizaje organizacional: Proceso de creación, transferencia y retención de conocimiento realizado para la generación de soluciones viables a problemas propios de las organizaciones (Cyert y March, 1992; Easterby-Smith, Crossan y Niccolini, 2000).

Entorno virtual de aprendizaje: Conjunto de aplicaciones y procesos basados en plataformas web, empleados para el entrenamiento, formación y capacitación virtual, generalmente utilizado dentro de las instituciones de educación (Weller, 2007).

Universidad corporativa: Unidad educacional, con carácter de herramienta estratégica, diseñada para asistir a las organizaciones en la consecución de su misión, mediante actividades que promueven el aprendizaje organizacional e individual (Allen, 2002).

\section{Revisión del marco referencial y Metodología de Clasificación}

El método de clasificación propuesto para los distintos artículos de investigación se realizó con las siguientes categorías relevantes acerca de la temática de aprendizaje organizacional y universidad corporativa, que tiene como puntos importantes los entornos virtuales de aprendizaje organizacional: (a) Lugar geográfico en el que se desarrolló la investigación/artículo, (b) Sector en el que se efectuó la investigación, (c) Temática Central de la Investigación, (d) Estrategia educativa, (e) Tecnologías de información relacionadas, (f) Enfoque de competencias y (g) Resultados obtenidos.

La categoría (a) representa el Lugar geográfico en el que se desarrolló del trabajo de investigación, teniendo en cuenta como ubicación fuente al continente y el país. Las sub.-categorías planteadas son las siguientes: AM: América (1: América del norte, 2: América Central, 3: América del Sur, 3.1: Colombia), AF: África, AS: Asia, MO: Medio Oriente, E: Europa, y OC: Oceanía. (Ver Tabla 1).
Tabla 1. Clasificación Variable (a): Lugar geográfico

\begin{tabular}{|c|c|}
\hline \multicolumn{2}{|c|}{$\begin{array}{l}\text { Variable (a): Lugar geográfico en el que se desarrolló del } \\
\text { trabajo investigativo }\end{array}$} \\
\hline América & AM \\
\hline Norte & $\mathrm{AM}, 1$ \\
\hline Central & $\mathrm{AM}, 2$ \\
\hline Sur & $\mathrm{AM}, 3$ \\
\hline Colombia & $\mathrm{AM}, 3.1$ \\
\hline África & $\mathrm{AF}$ \\
\hline Asia & AS \\
\hline Medio oriente & MO \\
\hline Europa & E \\
\hline Oceanía & OC \\
\hline
\end{tabular}

La categoría (b) presenta los distintos Sectores en el cual se aplicó el trabajo de investigación y las sub-categorías que se utilizaron para la descripción de la variable fueron: ED: Educación, IN: Industrial (1: Textil, 2 2: Automotriz, 3: Metalmecánica), SA: Salud, O: Otros. (Ver Tabla 2)

Tabla 2. Clasificación Variable (b): Sector Económico

\begin{tabular}{|c|c|}
$\begin{array}{c}\text { Variable (b): Sector en el que se centró del trabajo investi- } \\
\text { gativo }\end{array}$ \\
\hline Educación & ED \\
\hline Industrial & $\mathrm{IN}$ \\
\hline Tecnología & $\mathrm{IN}, 1$ \\
\hline Automotriz & $\mathrm{IN}, 2$ \\
\hline Metalmecánico & $\mathrm{IN}, 3$ \\
\hline Salud & $\mathrm{SA}$ \\
\hline Otros & $\mathrm{O}$ \\
\hline
\end{tabular}


En particular, en el caso del sector Educación se hace referencia a todos los modelos que no se validan o especifican que son aplicables a una industria en particular, sino que se llevan a cabo en instituciones educativas o de investigación; para el caso del Metalmecánico se refiere a aquellas aplicaciones que se dan en este sector de la economía en particular.

En la categoría (c), Temática central de la investigación, trata sobre los distintos frentes en los cuales ésta fue desarrollada. Las sub-categorías utilizadas fueron: SI: Sistemas integrales de aprendizaje organizacional como tema central, EV: Entornos virtuales de aprendizaje, UC: Universidad Corporativa, UE: Programas de Universidad empresa, TI: Tecnologías de Información, NE: No especificado (investigaciones abiertas en los que no se hace claridad de la temática central). Ver Tabla 3.

Tabla 3. Clasificación Variable (c): Temática Central de la Investigación

\begin{tabular}{|l|c|}
\hline \multicolumn{2}{|c|}{ Variable (c): Temática Central de la Investigación } \\
\hline $\begin{array}{l}\text { Sistemas integrales de aprendizaje organiza- } \\
\text { cional }\end{array}$ & $\mathrm{SI}$ \\
\hline Entornos virtuales de aprendizaje & $\mathrm{EV}$ \\
\hline Universidad Corporativa & $\mathrm{UC}$ \\
\hline Programas de Universidad empresa & $\mathrm{UE}$ \\
\hline Tecnologías de Información & $\mathrm{TI}$ \\
\hline & $\mathrm{NE}$ \\
\hline
\end{tabular}

La categoría (d) representa todas las estrategias de educación planteadas en los trabajos de investigación relacionados con las temáticas objeto de estudio. Las sub-categorías utilizadas en este caso fueron las siguientes: PC: Programas de Capacitación, PV: Programas de enseñanza virtual, PP: Programas presenciales en centros de educación superior, $\mathbf{H}$ : Programas Híbridos de desarrollo educativo. (Ver Tabla 4)
Tabla 4. Clasificación Variable (d): Estrategias de Educación

\begin{tabular}{|l|c|}
\hline \multicolumn{2}{|c|}{ Variable (d): Estrategias de Educación } \\
\hline Programas de Capacitación & PC \\
\hline Programas de enseñanza virtual & PV \\
\hline $\begin{array}{l}\text { Programas presenciales en centros de } \\
\text { educación superior }\end{array}$ & PP \\
\hline \\
Programas Híbridos
\end{tabular}

La categoría (e), Tecnologías de información, se refiere al uso de TIC's en el proceso del desarrollo educativo. Las subcategorías utilizadas fueron las siguientes: VP: Plataformas Virtuales, PI: Programas ejecutados en la intranet, PT: Plataformas en telefonía celular, NE: No especificado (Ver Tabla 5).

Tabla 5. Clasificación Variable (d): Tecnologías de información

\begin{tabular}{|l|c|}
\hline \multicolumn{2}{|c|}{ Variable (e): Tecnologías de información } \\
\hline Plataformas Virtuales & VP \\
\hline Programas ejecutados en la intranet & $\mathrm{PI}$ \\
\hline Plataformas en telefonía celular & $\mathrm{PT}$ \\
\hline No especificado & $\mathrm{NE}$ \\
\hline
\end{tabular}

La categoría (f), Enfoque de competencias, se basa en el énfasis de fortalecimiento educativo de los programas o sistemas mencionados, ya que algunos de ellos abarcan aspectos específicos de una organización. Por lo tanto, la clasificación realizada se presentó de la manera siguiente: CS: Competencias del ser, se basan más en programas dirigidos a fortalecer comportamiento necesarios para la ejecución de un puesto de trabajo o identificarse con la organización; CT: Competencias Técnicas, se refiere a como se realizan las actividades básicas en la ejecución de un puesto de trabajo; CO: Competencias organizacionales, apuntan a la misión y visión (Ver Tabla 6). 
Tabla 6. Clasificación Variable (f): Enfoque de competencias

\begin{tabular}{|l|c|}
\hline \multicolumn{2}{|c|}{ Variable (f): Enfoque de competencias } \\
\hline Competencias del ser & $\mathrm{CS}$ \\
\hline Competencias Técnicas & $\mathrm{CT}$ \\
\hline Competencias Organizacionales & $\mathrm{CO}$ \\
\hline
\end{tabular}

Una de las variables más utilizadas son los resultados obtenidos; es decir, la categoría (h), la cual considera el caso de la consecución de los objetivos planteados por la investigación, teniendo en cuenta las conclusiones del trabajo. Se denotan así: 0: Objetivos No logrados, 1: Objetivos logrados (Ver Tabla 7).

Tabla 7. Clasificación Variable (g): Resultados

\begin{tabular}{|l|c|}
\hline \multicolumn{2}{|c|}{ Variable (g): Resultados } \\
\hline Objetivo Logrado & 1 \\
\hline Objetivo No logrado & 0 \\
\hline
\end{tabular}

Además de la clasificación presentada para los aspectos más importantes en los trabajos de investigación consultados, se realizó la identificación de aquellas publicaciones que tocan aspectos relacionados a los sistemas integrales de aprendizaje en las organizaciones (Ver Tabla 8).

Tabla 8. Ejemplos de Artículos del SIAO

\begin{tabular}{|l|l|}
\hline \multirow{2}{*}{ Artículos Relacionados con la temática } \\
\hline \multirow{3}{*}{ SIAO } & $\begin{array}{l}\text { Virtual learning: emerging trend in education sector } \\
\text { of Pakistan (2013) }\end{array}$ \\
\cline { 2 - 3 } & $\begin{array}{l}\text { Estrategias de participación e interacción en entor- } \\
\text { nos virtuales de aprendizaje (2012) }\end{array}$ \\
\cline { 2 - 2 } & $\begin{array}{l}\text { Proyectos de vinculación escuela-empresa como } \\
\text { estrategia de apoyo en la calidad del proceso } \\
\text { enseñanza aprendizaje de la educación superior } \\
(2012)\end{array}$ \\
\hline
\end{tabular}

\section{Tabulación de la información por categorías de clasificación}

La Tabla 9 muestra el resumen de la clasificación realizada al revisar la literatura, conforme a las categorías definidas.

Tabla 9. Tabla de clasificación de los Artículos del SIAO

\begin{tabular}{|c|c|c|c|c|c|c|c|c|c|}
\hline \multicolumn{10}{|c|}{ CLASIFICACION DE LAS INVESTIGACIONES } \\
\hline Año & Articulo & Revista & (A) & (B) & (C) & (D) & (E) & (F) & (G) \\
\hline 1991 & $\begin{array}{l}\text { Organizational Learning Curves: A } \\
\text { method for Investigating Intra-plant } \\
\text { Transfer of Knowledge Acquired } \\
\text { through Learning by Doing }\end{array}$ & $\begin{array}{l}\text { Organization } \\
\text { Science }\end{array}$ & $\mathrm{AM}, 1$ & $\mathrm{IN}, 1$ & SI & PC & NE & CT & 1 \\
\hline 1992 & $\begin{array}{l}\text { Organizational Learning: A review } \\
\text { of the literature with implcations for } \\
\text { HRD Professionals }\end{array}$ & $\begin{array}{l}\text { Human Re- } \\
\text { source Devel- } \\
\text { opment Quar- } \\
\text { terly }\end{array}$ & NE & NE & NE & NE & NE & NE & 1 \\
\hline 1997 & $\begin{array}{l}\text { Developing a Robust Model of the } \\
\text { Virtual Corporate University }\end{array}$ & $\begin{array}{l}\text { Journal of } \\
\text { Knowle dge } \\
\text { Management }\end{array}$ & & $\mathrm{IN}, 1$ & UC & PV & VP & & 1 \\
\hline 1998 & $\begin{array}{l}\text { Innovation, Market Orientation, and } \\
\text { Organizational Learning: An Integra- } \\
\text { tion and Empirical Examination }\end{array}$ & $\begin{array}{l}\text { Journal of Mar- } \\
\text { keting }\end{array}$ & $\mathrm{AM}, 1$ & $\mathrm{O}$ & SI & NE & NE & NE & 1 \\
\hline 1998 & Corporate Universities of the future & $\begin{array}{l}\text { Career devel- } \\
\text { opment inter- } \\
\text { national }\end{array}$ & $\mathrm{AM}, 1$ & $\begin{array}{l}E \mathrm{D} \\
\mathrm{IN}\end{array}$ & $\begin{array}{l}\text { U C } \\
\text { UE }\end{array}$ & $\begin{array}{l}\text { P C } \\
\text { PV }\end{array}$ & $\mathrm{NE}$ & $\begin{array}{l}\mathrm{CS}, \mathrm{CT} \\
\mathrm{CO}\end{array}$ & 1 \\
\hline
\end{tabular}




\begin{tabular}{|c|c|c|c|c|c|c|c|c|c|}
\hline 1999 & $\begin{array}{l}\text { Structuring for Organizational learn- } \\
\text { ing }\end{array}$ & $\begin{array}{l}\text { The learning } \\
\text { Organization }\end{array}$ & E & IN & SI & NE & NE & NE & 1 \\
\hline 2000 & $\begin{array}{l}\text { Case research into corporate univer- } \\
\text { sity developments }\end{array}$ & $\begin{array}{l}\text { Journal of } \\
\text { Workp I a c e } \\
\text { Learning }\end{array}$ & E & IN & UC & & NE & & 1 \\
\hline 2001 & $\begin{array}{l}\text { On differences between organization- } \\
\text { al learning and learning organization }\end{array}$ & $\begin{array}{l}\text { The learning } \\
\text { organization }\end{array}$ & $E$ & IN & NE & & & & 1 \\
\hline 2001 & $\begin{array}{l}\text { Configuring the corporate universi- } \\
\text { ty - managing a portfolio of thinking } \\
\text { schools }\end{array}$ & $\begin{array}{l}\text { Journal of } \\
\text { workp l a c e } \\
\text { learning }\end{array}$ & $E$ & & $\begin{array}{l}\text { U C } \\
\text { UE }\end{array}$ & & & $\begin{array}{l}\mathrm{CS}, \mathrm{CT} \\
\mathrm{CO}\end{array}$ & 1 \\
\hline 2001 & $\begin{array}{l}\text { How to configure the corporate uni- } \\
\text { versity for success }\end{array}$ & $\begin{array}{l}\text { Journal of } \\
\text { Workplace } \\
\text { Learning }\end{array}$ & $E$ & ED & UC & $\mathrm{H}$ & NE & СT & 1 \\
\hline 2002 & $\begin{array}{l}\text { Corporate Universitites vs higher ed- } \\
\text { ucation institutions }\end{array}$ & $\begin{array}{l}\text { Industrial and } \\
\text { commercial } \\
\text { training }\end{array}$ & $\mathrm{AM}, 1$ & IN & $\begin{array}{l}\text { E V } \\
\text { U C } \\
\text { U E } \\
\quad T I\end{array}$ & $\mathrm{H}$ & $\begin{array}{l}\text { V P } \\
\mathrm{PI}\end{array}$ & $\begin{array}{l}\mathrm{CS}, \mathrm{CT} \\
\mathrm{CO}\end{array}$ & 1 \\
\hline 2002 & $\begin{array}{l}\text { Managing the corporate university } \\
\text { learning curve }\end{array}$ & $\begin{array}{l}\text { Journal of } \\
\text { workp l a c e } \\
\text { learning }\end{array}$ & $E$ & $\begin{array}{l}E \mathrm{D} \\
\text { IN }\end{array}$ & UC & & & & 1 \\
\hline 2002 & $\begin{array}{l}\text { Corporate universities - an analytical } \\
\text { framework }\end{array}$ & $\begin{array}{l}\text { Journal of } \\
\text { Management } \\
\text { Development }\end{array}$ & $E$ & $\mathrm{O}$ & UC & $\mathrm{H}$ & NE & $\begin{array}{l}\mathrm{CT}, \mathrm{CO} \\
\mathrm{CS}\end{array}$ & 1 \\
\hline 2002 & $\begin{array}{l}\text { Managing the corporate university } \\
\text { watershed }\end{array}$ & $\begin{array}{l}\text { Journal of } \\
\text { Workp I a c e } \\
\text { Learning }\end{array}$ & $E$ & $\mathrm{O}$ & UC & $\mathrm{H}$ & NE & $\begin{array}{l}\mathrm{CT}, \mathrm{CO} \\
\mathrm{CS}\end{array}$ & 1 \\
\hline 2005 & $\begin{array}{l}\text { Organizational learning capability: a } \\
\text { proposal of measurement }\end{array}$ & $\begin{array}{l}\text { Journal of } \\
\text { Business Re- } \\
\text { search }\end{array}$ & $E$ & IN & SI & NE & NE & NE & 1 \\
\hline 2005 & $\begin{array}{l}\text { Would the real corporate university } \\
\text { please stand up? }\end{array}$ & $\begin{array}{l}\text { Journal of Eu- } \\
\text { ropean Indus- } \\
\text { trial Training }\end{array}$ & E & $\mathrm{O}$ & UC & $\mathrm{H}$ & NE & $\mathrm{CO}$ & 1 \\
\hline 2005 & The corporate university & $\begin{array}{l}\text { Journal of Eu- } \\
\text { ropean Indus- } \\
\text { trial Training }\end{array}$ & $E$ & $\mathrm{O}$ & UC & $\mathrm{H}$ & NE & $\mathrm{CO}$ & 1 \\
\hline 2005 & $\begin{array}{l}\text { Strategy by degrees: How corporate } \\
\text { universities can help companies plan } \\
\text { for the future }\end{array}$ & $\begin{array}{l}\text { Development } \\
\text { and Learning in } \\
\text { Organizations: } \\
\text { An Internation- } \\
\text { al Journal }\end{array}$ & $E$ & $\mathrm{O}$ & UC & $\mathrm{H}$ & NE & $\mathrm{CO}$ & 1 \\
\hline
\end{tabular}




\begin{tabular}{|c|c|c|c|c|c|c|c|c|c|}
\hline 2005 & $\begin{array}{l}\text { The rise and rise of the corporate uni- } \\
\text { versity }\end{array}$ & $\begin{array}{l}\text { Journal of Eu- } \\
\text { ropean Indus- } \\
\text { trial Training }\end{array}$ & E & ED & UC & & & & 1 \\
\hline 2005 & E\#learning in the corporate university & $\begin{array}{l}\text { Journal of Eu- } \\
\text { ropean Indus- } \\
\text { trial Training }\end{array}$ & E & IN & EV & PV & NE & & 1 \\
\hline 2006 & $\begin{array}{l}\text { Corporate universities: a catalyst for } \\
\text { strategic human resource develop- } \\
\text { ment }\end{array}$ & $\begin{array}{l}\text { Journal of Eu- } \\
\text { ropean Indus- } \\
\text { trial Training }\end{array}$ & OC & IN & UC & & & & 1 \\
\hline 2006 & $\begin{array}{l}\text { The corporate university's role in } \\
\text { managing an epoch in learning or- } \\
\text { ganisation innovation }\end{array}$ & $\begin{array}{l}\text { Journal of } \\
\text { Workp I a c e } \\
\text { Learning }\end{array}$ & $E$ & IN & UC & $\mathrm{H}$ & NE & CS - CT & 1 \\
\hline 2007 & $\begin{array}{l}\text { Developing a qualitative universi- } \\
\text { ty-corporate education understand- } \\
\text { ing of partnerships }\end{array}$ & $\begin{array}{l}\text { Management } \\
\text { Decision }\end{array}$ & OC & O & UC & $\mathrm{H}$ & NE & $\begin{array}{l}\text { CT, CO, } \\
\quad \text { CS }\end{array}$ & 1 \\
\hline 2007 & $\begin{array}{l}\text { Diversity learning, knowledge diversi- } \\
\text { ty and inclusion }\end{array}$ & $\begin{array}{l}\text { Equal Opportu- } \\
\text { nities Interna- } \\
\text { tional }\end{array}$ & E & $\mathrm{O}$ & UC & $\mathrm{H}$ & NE & $\begin{array}{l}\mathrm{CT}, \mathrm{CO} \\
\mathrm{CS}\end{array}$ & 1 \\
\hline 2007 & $\begin{array}{l}\text { An exploratory study of corporate uni- } \\
\text { versities in China }\end{array}$ & $\begin{array}{l}\text { Journal of } \\
\text { Workpla c e } \\
\text { Learning }\end{array}$ & AS & $\mathrm{O}$ & UC & $\mathrm{H}$ & NE & $\begin{array}{l}\text { CT, CO, } \\
\quad \text { CS }\end{array}$ & 1 \\
\hline 2007 & $\begin{array}{l}\text { The corporate university landscape in } \\
\text { Germany }\end{array}$ & $\begin{array}{l}\text { Journal of } \\
\text { Workp I a c e } \\
\text { Learning }\end{array}$ & $E$ & $\mathrm{O}$ & UC & & & & 1 \\
\hline 2008 & $\begin{array}{l}\text { Investigating organizational learning } \\
\text { in eGovernment projects: A multi-the- } \\
\text { oretic approach }\end{array}$ & $\begin{array}{l}\text { Strategic Infor- } \\
\text { mation Siste- } \\
\text { mas }\end{array}$ & AS & ED & $\mathrm{Si}, \mathrm{TI}$ & NE & NE & NE & 1 \\
\hline 2008 & $\begin{array}{l}\text { Universidade corporativa:espaço } \\
\text { diferenciado de apredizagem no tra- } \\
\text { balho }\end{array}$ & Pensam. Real. & $\mathrm{AM}, 3$ & $\begin{array}{l}E \mathrm{D} ; \\
\mathrm{IN}\end{array}$ & UC & $(-)$ & $(-)$ & CS; CT & 1 \\
\hline 2009 & $\begin{array}{l}\text { Aviva España: Universidad corpora- } \\
\text { tiva }\end{array}$ & $\begin{array}{l}\text { Capital Huma- } \\
\text { no }\end{array}$ & E & $\mathrm{O}$ & $\begin{array}{l}\text { U C } \\
\text { UE }\end{array}$ & $\begin{array}{l}\text { P C } \\
\text { PV }\end{array}$ & NE & $\begin{array}{l}\text { CT, CO, } \\
\text { CS }\end{array}$ & 1 \\
\hline 2009 & $\begin{array}{l}\text { Gestión de un entorno visrtual de } \\
\text { aprendizaje para el desarrollo de } \\
\text { competencias profesionales inter- } \\
\text { culturales: una experiencia de edu- } \\
\text { cación superior }\end{array}$ & Apertura & $E$ & ED & EV & PV & VP & $\mathrm{CS} ; \mathrm{CT}$ & 1 \\
\hline 2009 & $\begin{array}{l}\text { Exploring the growing phenomenon } \\
\text { of university-corporate education } \\
\text { partnerships }\end{array}$ & $\begin{array}{l}\text { Management } \\
\text { Decision }\end{array}$ & $\begin{array}{l}\text { A M } \\
1 ; \quad E ; \\
O C\end{array}$ & $\begin{array}{l}E D \\
\text { IN }\end{array}$ & UE & $\begin{array}{c}\mathrm{P} \mathrm{C} \\
\mathrm{H}\end{array}$ & $\begin{array}{l}\text { VP } \\
\text { PI, }\end{array}$ & $\begin{array}{l}\text { CS, CT, } \\
\text { CO }\end{array}$ & 1 \\
\hline
\end{tabular}




\begin{tabular}{|c|c|c|c|c|c|c|c|c|c|}
\hline 2009 & $\begin{array}{l}\text { Corporate universities in China: pro- } \\
\text { cesses, issues and challenges }\end{array}$ & $\begin{array}{l}\text { Journal of } \\
\text { Workp I a c e } \\
\text { Learning }\end{array}$ & AS & $\mathrm{O}$ & UC & $\mathrm{H}$ & NE & $\begin{array}{l}\text { CT, } \mathrm{CO} \\
\mathrm{CS}\end{array}$ & 1 \\
\hline 2010 & $\begin{array}{l}\text { Trainingevaluation in italian corpo- } \\
\text { rate universities: a stakeholder based } \\
\text { analysis }\end{array}$ & $\begin{array}{l}\text { Internation- } \\
\text { al Journal of } \\
\text { training and } \\
\text { development }\end{array}$ & E & $\begin{array}{l}\text { E D } \\
\text { IN }\end{array}$ & UC & $\mathrm{H}$ & VP & $\begin{array}{l}\mathrm{CT}, \mathrm{CO} \\
\mathrm{CS}\end{array}$ & 1 \\
\hline 2011 & $\begin{array}{l}\text { Un modelo de control de gestión ha- } \\
\text { cia el aprendizaje organizacional }\end{array}$ & $\begin{array}{l}\text { Dimensión em- } \\
\text { presarial }\end{array}$ & AM, 3 & IN & SI & NE & NE & NE & 1 \\
\hline 2011 & $\begin{array}{l}\text { Developing simulations in multi-us- } \\
\text { er virtual environmentes to enhance } \\
\text { healthcare education }\end{array}$ & $\begin{array}{l}\text { British Journal } \\
\text { of educational } \\
\text { technology }\end{array}$ & OC & $\begin{array}{l}\mathrm{SA} \\
\mathrm{ED}\end{array}$ & $\begin{array}{l}\text { E V ; } \\
\text { PV }\end{array}$ & $\mathrm{H}$ & VP & CT & 1 \\
\hline 2011 & $\begin{array}{l}\text { Competencias en los procesos de } \\
\text { enseñanza aprendizaje virtual y sem- } \\
\text { ipresencial }\end{array}$ & $\begin{array}{l}\text { Revista científi- } \\
\text { ca de Educo- } \\
\text { municación }\end{array}$ & E & ED & EV & PV & VP & CS; CT & 1 \\
\hline 2011 & $\begin{array}{l}\text { Empresas de base tecnológicas } \\
\text { derivadas de la universidad: el vín- } \\
\text { culo empresario - oportunidad tec- } \\
\text { nológica }\end{array}$ & $\begin{array}{l}\text { Revista de ad- } \\
\text { ministraçao e } \\
\text { inovaçao }\end{array}$ & E & $\begin{array}{l}\text { E D } \\
\text { IN }\end{array}$ & UC & & & $\begin{array}{l}\text { CT, } \mathrm{CO} \\
\mathrm{CS}\end{array}$ & 1 \\
\hline 2011 & $\begin{array}{l}\text { Punto de inflexión entre empresas y } \\
\text { universidades ante la relación Uni- } \\
\text { versidad, empresa y estado en Co- } \\
\text { lombia }\end{array}$ & Univ. Empresa & $\begin{array}{r}\mathrm{A} M \\
3.1\end{array}$ & $\begin{array}{l}\text { E D } \\
\text { IN }\end{array}$ & UC & & & CS; CT & 1 \\
\hline 2012 & $\begin{array}{l}\text { Exploring the Corporate University } \\
\text { Phenomenon: Developmen tand Im- } \\
\text { plementation of a Comprehensive } \\
\text { Survey }\end{array}$ & $\begin{array}{l}\text { Human Re- } \\
\text { source Devel- } \\
\text { opment Quar- } \\
\text { terly }\end{array}$ & $\mathrm{AM}, 1$ & NE & UC & NE & NE & NE & 1 \\
\hline 2012 & $\begin{array}{l}\text { Estrategias de participación e in- } \\
\text { teracción en entronos virtuales de } \\
\text { aprendizaje }\end{array}$ & Anagramas & $\begin{array}{c}\mathrm{A} M \\
3.1\end{array}$ & ED & $\begin{array}{l}\mathrm{E} \mathrm{V} \\
\mathrm{TI}\end{array}$ & $\begin{array}{l}\mathrm{P} V \\
\mathrm{PP}\end{array}$ & $\begin{array}{l}\text { V P ; } \\
\text { PT }\end{array}$ & CS; CT & 1 \\
\hline 2012 & $\begin{array}{l}\text { Proyectos de vinculación escue- } \\
\text { la-empresa como estrategia de apoyo } \\
\text { en la calidad del proceso enseñanza } \\
\text { aprendizaje de la educación superior }\end{array}$ & $\begin{array}{l}\text { Revista inter- } \\
\text { nacional ad- } \\
\text { ministración y } \\
\quad \text { finanzas }\end{array}$ & $\mathrm{AM}, 2$ & ED & UE & $\mathrm{H}$ & & CT & 1 \\
\hline 2012 & $\begin{array}{l}\text { Use of clinical cases in a virtual learn- } \\
\text { ing environment as an approach to } \\
\text { teaching human embryology }\end{array}$ & Int. J Morpho & $\mathrm{AM}, 3$ & $\begin{array}{l}\mathrm{SA} \\
\mathrm{ED}\end{array}$ & $\begin{array}{l}\text { E V } \\
\text { PV }\end{array}$ & $\mathrm{H}$ & VP & CT & 1 \\
\hline 2012 & $\begin{array}{l}\text { Aplicación de un ambiente virtual de } \\
\text { aprendizaje orientado a la formación } \\
\text { empresarial }\end{array}$ & $\begin{array}{l}\text { Estudios ger- } \\
\text { enciales }\end{array}$ & $\begin{array}{c}\mathrm{A} M \\
3.1\end{array}$ & ED & $\begin{array}{l}\mathrm{E} \mathrm{V} \\
\mathrm{TI}\end{array}$ & PV & VP & CS; CT & 1 \\
\hline 2012 & $\begin{array}{l}\text { Los modelos europeos de transferec- } \\
\text { nia de tecnología universidad-empre- } \\
\text { sa }\end{array}$ & $\begin{array}{l}\text { Revista de } \\
\text { e c o n o m í a } \\
\text { mundial }\end{array}$ & E & IN & $\begin{array}{l}\text { E V } \\
\text { UE }\end{array}$ & $\mathrm{H}$ & VP & CT & 1 \\
\hline
\end{tabular}




\begin{tabular}{|c|l|c|c|c|c|c|c|c|}
\hline 2012 & $\begin{array}{l}\text { Metodología para valoración para } \\
\text { proyectos de transferencia tecnológi- } \\
\text { ca universitaria caso aplicado - uni- } \\
\text { versidad de Antioquia }\end{array}$ & $\begin{array}{c}\text { rev. Fac. cienc. } \\
\text { Econ. }\end{array}$ & $\begin{array}{c}\text { A M , } \\
3.1\end{array}$ & $\begin{array}{c}\text { E D ; } \\
\text { IN }\end{array}$ & UC & CS; CT & 1 \\
\hline 2013 & $\begin{array}{l}\text { Virtual learning: emerging trend in } \\
\text { education sector of Pakistan }\end{array}$ & $\begin{array}{l}\text { Interdiscipli- } \\
\text { nary journal of } \\
\text { contemporary } \\
\text { research in } \\
\text { business }\end{array}$ & AS & ED & E V ; \\
TI V ; & PP & VP & CT \\
\hline & $\begin{array}{l}\text { The generation of public intellectuals: } \\
\text { Corporate Universities, graduate em- } \\
\text { ployees and the academic labor }\end{array}$ & $\begin{array}{c}\text { Labor studies } \\
\text { journal }\end{array}$ & AM, 2 & E D ; \\
IN & UC & & CS; CT \\
\hline
\end{tabular}

\section{Marco de referencia de los sis- temas de aprendizaje organi- zacional y las Universidades Corporativas}

Del análisis realizado en los anteriores apartados, se procedió a construir el marco de referencia sobre los sistemas de aprendizaje organizacional y de las universidades corporativas, resaltando aquellos trabajos que proporcionan una base conceptual y casos aplicados de dichas temáticas. En las siguientes secciones se muestran los autores e investigaciones destacadas, mencionando sus principales aportes y conclusiones.

\subsection{Entornos virtuales de aprendizaje}

Los avances en las TIC's han facilitado el acceso a la información y conocimiento de personas y organizaciones en general. Esto ha permitido que los métodos convencionales de aprendizaje le cedan el paso a plataformas virtuales, las cuales pueden operar de manera asíncrona y con mayor cobertura, pudiendo ser accedidas en cualquier lugar del mundo.

La educación superior por medio de plataformas virtuales es uno de los ejemplos de la migración de las metodologías convencionales hacia las TIC's. Actualmente, se ofrecen entrenamiento y formación virtuales incluyendo tanto programas de pregrado como de postgrado, que pueden ser cursados manteniendo los mismos estándares de calidad que su contraparte presencial.

No obstante, puede considerarse que el aprendizaje virtual entrega beneficios distintos a las metodologías tradicionales ya que fortalecen otro tipo de competencias y permiten un acceso rápido a la información. Ibernón, Silva y Guzmán (2011) muestran por medio de una investigación cualitativa cómo se fortalecieron las competencias de estudiantes y profesores en el manejo de tecnologías de información y aparatos electrónicos, en busca de una estandarización de los sistemas educativos en Europa. Hasta el año de la publicación, el $78 \%$ de los educadores implementaban algún tipo de herramienta electrónica y se esperaba que con el fortalecimiento de estas competencias se alcanzara un mayor nivel de desempeño.

En el caso de la educación europea, el e-learning no constituye un sistema estático; por el contrario, el fortalecimiento de las competencias pretende abrir fronteras de información que permitan un desarrollo de la educación y la investigación. Un estudio plantea un proyecto para el fortalecimiento bipartita entre España y México, en el cual el aprendizaje virtual se genera en el apoyo mutuo entre estudiantes en busca de robustecer competencias, lo cual es enriquecedor no solo para el proceso de generación de conocimiento sino para el desarrollo, incluso a nivel cultural, por medio de la integración (Farías y Montoya, 2009).

Las empresas pueden encontrar en los entornos virtuales de aprendizaje herramientas altamente funcionales. En un acercamiento al aprendizaje en las empresas, Reinoso (2012) realizó una metodología de aprendizaje dirigida a desarrollar competencias de emprendimiento en los jóvenes, es decir la utilidad en este caso específico es para la educación superior, pero que puede impactar a las organizaciones. El trabajo se basó en el diseño de una plataforma virtual llamada Dokeos aplicada a 22 estudiantes escogidos por conocimientos y aptitudes necesarias, a quienes se les dicto un curso de emprendimiento y creación de empresas con resultados positivos.

Rogers (2011) presenta en su investigación un desarrollo en entornos virtuales de aprendizaje que apoya las prácticas médicas. El trabajo consistió en el monta- 
je de una plataforma virtual en la cual se tenía acceso a las historias médicas de los pacientes; adicionalmente, estudiantes de medicina y enfermería tomaban decisiones relevantes con impacto sobre los resultados de los procedimientos de salud. Esta investigación se basaba en metodologías grupales de trabajo colaborativo. Sin embargo, se evidencian algunas limitaciones en cuanto al desarrollo de información especializada, tanto a la expuesta por el caso médico como a la presentada por los estudiantes.

También en el área de la salud, Ginani, Gadelha y Augusto (2012) presentan una investigación realizada alrededor del aprendizaje virtual en embriología. El trabajo consistió en una prueba comparativa entre dos grupos de estudiantes de medicina a quienes les fueron presentados casos clínicos por medio de blogs, permitiéndoles presentar sus comentarios y observaciones de tipo científico. Posteriormente, fueron evaluados los conocimientos adquiridos por ambos grupos y los resultados obtenidos fueron favorables en ambos.

Las investigaciones anteriores muestran la amplitud en cuanto a la investigación relacionada con los entornos virtuales de aprendizaje en el sector educativo. No obstante, también existen diversas investigaciones desarrolladas en el ámbito organizacional. Ejemplo de ello es el caso presentado por Aceytuno y Caceres (2012), quienes analizan cómo son y qué resultados entregan las relaciones entre las empresas y las instituciones de educación superior, resaltando que el desarrollo de alianzas entre ellas es fundamental y beneficioso para ambas.

En el siguiente apartado se presenta el concepto de universidad corporativa, el cual se muestra como un mecanismo de aprendizaje híbrido, desarrollado en el interior de las organizaciones de la mano, en muchos casos, de instituciones educativas de distintos niveles.

\subsection{Universidad Corporativa}

Las universidades corporativas se han convertido en un escenario colaborativo entre las empresas y las instituciones de educación, el cual permite el desarrollo de diversas actividades de capacitación, entrenamiento y formación (Guerci, Bartezzagui y Solari, 2010). Las universidades corporativas representan un paso importante hacia la construcción de sistemas integrales de aprendizaje en las organizaciones mediante los cuales es posible el desarrollo de los distintos tipos de competencias (organizacionales, de área y técnicas). En este sentido, Gilbert (2013) expone que el fenómeno de las universidades corporativas apoya el desarrollo del personal como una herramienta para el fortalecimiento de organizaciones públicas y privadas.

Abel y Li (2012) exploran y hacen avanzar la comprensión del fenómeno de universidad corporativa. Los autores realizan una encuesta sobre las universidades corporativas para obtener una perspectiva sobre los procesos importantes en estas. Un total de 210 universidades corporativas participaron en el estudio en la región de América del Norte; los resultados revelaron cinco procesos importantes de las universidades corporativas basadas en un análisis factorial: la alineación y la ejecución; el desarrollo de habilidades que apoyan las necesidades del negocio, utilizando la tecnología para apoyar la función de aprendizaje; el aprendizaje; la evaluación del desempeño, y la colaboración con el mundo académico.

Desde la perspectiva de un sistema integral de aprendizaje organizacional, la relación entre la universidad y la empresa se centra en los aspectos relacionados con el aprendizaje mismo. No obstante, como actividad generadora de conocimiento se encuentra también la investigación, la cual no solo implica la necesidad de gestionarlo sino que requiere de la colaboración mutua de empresas e instituciones de educación para conseguir el mejoramiento continuo (Gutiérrez y Berrio, 2011). De otra parte, se puede afirmar que la transferencia tecnológica constituye un camino para el desarrollo del aprendizaje conjunto entre las empresas y las organizaciones; por ejemplo, algunas instituciones educativas procuran el desarrollo de la transferencia tecnológica como escenario de colaboración, tal y como lo plantea Correa, Arango y Álvarez (2012).

Dealtry (2001) realiza su investigación con base en dos hipótesis acerca de si la empresa mejoraría su efectividad de una manera rápida o si podía optimizar su crecimiento y calidad implementando universidades corporativas; el autor concluye que ésta sí tiene las capacidades para desarrollar a aquella dada su capacidad de permitir el desarrollo de competencias.

Campos y Otros (2011), presentan una investigación sobre los spin-off de empresas que se generan a partir de las universidades y sus investigaciones, lo cual enriquece no solo los procesos organizacionales de cada institución sino a los estudiantes, quienes pueden promover sus competencias a nivel práctico a través del emprendimiento. Por su parte, Stumpf (1998) plantea en su investigación que las universidades corporativas, como toda unidad dentro de la empresa, tiene clientes y grupos de interés. Las universidades corporativas operan en un ambiente sujeto a tendencias demográficas, tecnológicas o políticas que puedan afectar su negocio. Sugiere que la universidad corporativa debe 
ser gestionada como una línea de negocio destinada a satisfacer necesidades internas de aprendizaje, y de vez en cuando externas, del personal de la compañía.

Ryan (2009) en su investigación tiene como objetivo establecer las razones que tienen las corporaciones para formar alianzas con las universidades y el rol de los programas de educación corporativa. Setenta y nueve líderes de alianzas entre corporaciones y universidades fueron analizados para realizar esta investigación, la cual concluyó que la principal razón para establecer este tipo de vínculos es la posibilidad que se les da de formar internamente a sus empleados y obtener un certificado de una universidad reconocida.

De acuerdo a Dealtry (2002), la universidad corporativa debe apuntarle a tener un efecto muy práctico en ayudar a los trabajadores a realizar sus ambiciones; esta debe conectar a los trabajadores con sus motivaciones y su deseo de lograr una mejor manera de trabajar y tener las oportunidades de pensar inteligentemente en un ambiente de aprendizaje seguro.

Ryan (2007) plantea una ayuda a las empresas para que tengan una estructura exitosa en sus programas de universidad corporativa, a través del análisis de variables fundamentales identificadas por medio de entrevistas cualitativas realizadas a 6 gerentes de empresas de distintos sectores y 3 gestores universitarios que toma como modelo.

Similarmente, Andresen y Lichtenberger (2007) establecen en su artículo de investigación que las universidades corporativas están teniendo un crecimiento importante debido a la necesidad que tienen las organizaciones en la gestión efectiva del conocimiento; por lo tanto, en la investigación se pretende mostrar un punto de vista frente a las universidades corporativas existentes y se llega a la conclusión de una necesidad de diversificación en el conocimiento por parte de las empresas.

Prince y Stewart (2002) contribuyen con un marco de referencia a partir de los conceptos importantes de la gestión del conocimiento, aprendizaje organizacional y organización de aprendizaje. Como conclusión, los autores aseguran que "el éxito de las universidades corporativas del futuro podría depender de su capacidad para gestionar y aprovechar la compleja interacción de los subsistemas de aprendizaje organizacional y menos en su capacidad para gestionar los programas de formación y educación".

De otro lado, la investigación de Walton (2005) tiene como propósito identificar diferencias y similitudes entre universidades tradicionales y corporativas, con el fin de visualizar resultados futuros de cada una de las aplicaciones específicas en su contexto. Shaw (2005) estudia en su artículo el caso exitoso de la universidad corporativa de Motorola en el Oriente y cómo esta logro traspasar fronteras, gracias al éxito en la gestión del conocimiento en las distintas instituciones que replicaron el modelo.

Dealtry (2002) busca estudiar la gestión del conocimiento en las universidades corporativas, durante momentos de crisis, lo cual puede aportar un valorar fundamental en entornos tan cambiantes como los de las organizaciones actuales inmersas en un mundo global y cambiante. Así mismo, en otra investigación (Dealtry, 2000) describe una metodología de investigación para evaluar la efectividad del desarrollo de universidades corporativas. Evaluada en diferentes compañías, la metodología ha demostrado ser una buena guía para la revisión y evaluación de universidad corporativa, aunque es claro que toma tiempo identificar el verdadero potencial de esta.

De manera similar, este autor (Dealtry, 2001) también recopila información sobre ideas y procesos que puedan generar una perspectiva distinta sobre el concepto de universidad corporativa incorporando propiedades de las escuelas de pensamiento para generar desarrollo estratégico en la empresa. También presenta en su investigación un resumen de proyectos de aprendizaje estratégico y con ello pretende dar una visión amplia del rol que ha tenido la universidad corporativa como un proceso de innovación dentro de una organización de aprendizaje (Dealtry, 2006).

Por su parte, Sham (2007) describe cómo ha sido la evolución de las universidades corporativas en China y cómo estas han surgido y evolucionado desde una perspectiva netamente práctica. Xuejun (2009) también estudia a fondo el estado del arte de las Universidades Corporativas en China, centrándose en el estudio de "los procesos y prácticas de las universidades corporativas en China, y descubrir los problemas y desafíos involucrados en la construcción y funcionamiento" de estas en dicho país.

Andersen (2007) realiza una revisión sobre el desarrollo de la universidad corporativa en Alemania en los últimos 15 años, comparando el modelo actual con el que se utiliza en Estados Unidos, evaluando niveles educativos, dirección estratégica, alianzas y acreditaciones entre otros, generando un modelo que puede plantear una solución a los retos de ese país.

De otro lado, Blass (2005) investiga sobre el crecimiento de la universidad corporativa y la competencia que ha generado a las universidades tradicionales. 
En el estudio se analizan las primeras con respecto a la posibilidad de darles poder para otorgar títulos, las diferencias con el aprendizaje tradicional y las condiciones que podrían favorecer su futuro.

Macpherson y Homan (2005) exploran el desarrollo del e-learning dentro de las universidades corporativas con el objetivo de detectar áreas necesarias para fortalecer nuevos procesos de aprendizaje organizacional, teniendo en cuenta aspectos pedagógicos y la capacidad de respuesta del alumno ante esta herramienta.

Holland y Pyman (2006) estudian el concepto de universidad corporativa como una plataforma estratégica clave para aumentar la ventaja competitiva organizacional. Analizando un caso de estudio, fue posible mostrar la relación que debe existir entre la universidad corporativa y los objetivos estratégicos para generar una ventaja competitiva.

Sandelands (1997) desarrolla un modelo de universidad corporativa virtual teniendo en cuenta problemas como la necesidad de entender el proceso y desarrollar una plataforma con la información adecuada, generar el soporte necesario y entender que se requiere mejorar el desempeño y el mejoramiento.

Como ejemplo de universidad corporativa en el contexto español está lo expuesto por Rodríguez (2009), quien muestra el caso de un modelo para su creación, desarrollado para asegurar el crecimiento profesional del personal en las organizaciones. El modelo propuesto cuenta con 4 facultades: 1) Facultad de negocio, 2) facultado técnica, 3) facultad de dirección y, por último, 4) centro de conocimiento. Uno de los principales objetivos de este programa es que los empleados sepan cuál es su itinerario formativo y cómo se prevé que evolucione a varios años.

En síntesis, se encontró que la universidad corporativa es una tendencia de aprendizaje en Europa, América del Norte y América Latina en los próximas años que, incluso, puede llevar a que estas unidades organizacionales sobrepasen en cantidad a las universidades tradicionales ya que las empresas querrán empleados con las capacidades y competencias necesarias para afrontar sus problemas particulares (Nixon y Helm, 2002; Haas y De Oliveira, 2008).

\subsection{Aprendizaje organizacional}

Según Hong (1999) la investigación sobre el aprendizaje organizacional se basa en el concepto de cómo aprende una organización o de aquello que ha aprendido una organización. Según el autor, los individu- os son los agentes para el aprendizaje y éste ocurre cuando los miembros de la organización detectan la discrepancia entre los resultados reales y los esperados, tratando de corregir los errores. Los nuevos conocimientos o "teoría en uso" están integrados en los modelos mentales compartidos por otros miembros de la organización o en los artefactos de la cultura, para que el aprendizaje se convierta en un proceso. Hay una suposición inherente en el concepto de aprendizaje organizacional que plantea que este mejora el rendimiento futuro a través del cambio de visión, nueva estructura organizativa, las nuevas acciones o la combinación de todo esto (Argyris y Schon, 1996).

Örtenblad (2001) plantea las diferencias que existen entre el aprendizaje organizacional y la organización de aprendizaje, concluyendo que el primer concepto hace referencia a procesos existentes dentro de la organización en relación al aprendizaje mientras que el segundo es una forma ideal de organización donde se plantea el aprendizaje como eje central.

Argyris y Schon (1978, 1996) establecen que el aprendizaje organizacional ocurre cuando los individuos dentro de una organización experimentan una situación problemática e investigan sobre ella en nombre de la organización. Estos padecen una sorprendente falta de coincidencia entre los resultados previstos frente a los reales y responden a la falta de coincidencia a través de un proceso de pensamiento y de acción adicional que les lleva a modificar su imagen de organización o de su comprensión de los fenómenos organizacionales, lo cual los lleva a la reestructuración de sus actividades, a fin de lograr resultados y expectativas, cambiando así la teoría organizacional en uso.

Hurley y Hult (1998) plantean que el aprendizaje organizacional y la orientación de mercados se direccionan hacia cómo las empresas se adaptan al ambiente en el cuál se desempeñan y cómo éstas, a su vez, logran crear ventaja competitiva. En su investigación los autores proponen la relación que existe entre innovación, orientación de mercado y aprendizaje organizacional, aplicando el estudio a una muestra de 9.648 empleados de 56 organizaciones en una agencia del gobierno de los Estados Unidos. Los resultados que arrojó el estudio fue que mayores niveles de innovación están relacionados con culturas que hacen énfasis en el aprendizaje organizacional, desarrollo y toma de decisiones participativa.

Según Epple, Argote y Devadas (1991), un modelo de curva de aprendizaje puede ser generalizado para investigar posibles comportamientos del aprendizaje organizacional. Los autores examinan la hipótesis de que los conocimientos adquiridos a través del aprendizaje 
en la práctica se materializaron en una organización de tecnología mediante el análisis de la cantidad de transferencia que se produce a través de cambios dentro de una planta de producción. Los métodos empleados en el estudio se ilustran mediante el análisis de los datos de una planta que comenzó la producción con un turno y después se añadió otro durante varios meses en el programa de producción. Si el conocimiento se incorpora en la tecnología, la transferencia a través de los cambios realizados debe ser completa, ya que dos turnos en la planta de producción utilizaron la misma tecnología. Los resultados indican que la transferencia de conocimiento se dio principalmente cuando el segundo turno fue introducido.

El aprendizaje organizacional, de acuerdo con Dixon (1992), es la competencia crítica destacada a partir de la década de los 90. La autora plantea que existen 3 fuerzas que impulsan a las compañías a integrar el proceso de aprendizaje organizacional: 1) el cambio en la naturaleza del trabajo, 2) el reto competitivo de la globalización y 3) el cambio inesperado en las organizaciones. En su artículo, la autora defiende esta última idea mediante el símil entre la teoría de Darwin y las compañías, en donde se dice que un organismo debe ser hábil de aprender a un ritmo igual o mayor al cambio que ocurre en su ambiente, por lo cual las empresas, como sistemas, deben incrementar su capacidad para aprender si quieren continuar operando en un ambiente caracterizado por cambios continuos.

Ortega y Locano (2011) proponen un modelo de control de gestión de cinco fases, orientado al aprendizaje organizacional. El modelo tiene como primera fase el diseño estratégico que rige toda la organización (misión, visión, objetivos y estrategias, etc); la segunda fase abarca la definición de los ejes, dimensiones e iniciativas que serán los derroteros o norte de la organización; la tercera fase corresponde al diseño de herramientas como los mapas estratégicos, presupuestos y cuadros de mando integral; la cuarta fase es de integración con los procesos a través de la cadena de valor y el sistema de gestión de calidad, para llegar a la fase final del sistema de información integral de gestión de la organización, donde se almacena toda historia, se registra el presente y se hace prospectiva.

De otra parte, Phang, Kankanhalli y Ang (2008) establecen que el aprendizaje organizacional es clave durante la implementación de un gobierno en línea (e-Government). Sin embargo, hay una falta de comprensión holística del aprendizaje organizacional en los cambios relacionados con las tecnologías de información $(\mathrm{TI})$ en las organizaciones públicas. Motivados por esas preocupaciones, los autores construyen un marco que interrelaciona los elementos pertinentes al cambio relacionados con TI desde cuatro perspectivas teóricas: la política de organización, cultura organizacional, teoría institucional y de organización de aprendizaje en sí. Interrelaciones entre los elementos de estas perspectivas son explicadas a través de las nociones de estructuras, modalidades y acciones de la teoría de la estructuración. Guiados por el marco, se realizó un estudio de caso para examinar el aprendizaje organizacional, el cual se produce durante un proyecto de administración electrónica con éxito. El marco propuesto ofrece dos ventajas teóricas y prácticas para comprender y facilitar el aprendizaje organizacional en los proyectos de administración electrónica.

Jerez, Cespedes y Valle (2003) proponen una escala de medición de la capacidad de aprendizaje de la organización, con el apoyo de los resultados de un estudio de validación que incluye una muestra de 111 empresas españolas de la industria química. Desde un punto de vista estratégico, la escala de medida identifica los elementos que forman la capacidad de aprendizaje, destacando su carácter complejo y multidimensional. La escala sugerida por los autores ofrece información que podría ser de utilidad para aquellos administradores que deseen mejorar la capacidad de aprendizaje en sus empresas.

Para Huber (1991) existen 4 componentes que se deben desarrollar con el fin de describir el proceso de aprendizaje: 1) la adquisición del conocimiento, 2) la distribución de la información, 3) la interpretación de la información y 4) la memoria organizacional, asumiendo que con este modelo de aprendizaje las organizaciones aprenden de una manera colectiva.

\section{Conclusiones}

Este artículo muestra la revisión de distintas publicaciones científicas acerca del aprendizaje organizacional, como proceso, y de las universidades corporativas, como iniciativa dirigida a proporcionar un mecanismo para la generación y transferencia de conocimiento. Dentro de los tópicos relacionados que abarca la revisión se encuentran los entornos virtuales de aprendizaje así como las universidades corporativas en distintos contextos. Se realizó la clasificación sistémica de las publicaciones de acuerdo a criterios como lugar y naturaleza del tema tratado.

En el actual estado del arte, el aprendizaje organizacional, como proceso, se entiende que surge de la necesidad de cambiar o de enfrentar el cambio frente a situaciones que requieren nuevas soluciones basadas en nueva información y conocimiento, bajo una dinámica que plantea el sostenimiento, e incluso in- 
corporación, de ventajas competitivas. Así mismo, los entornos de aprendizaje muestran tener distintas aplicaciones dentro y fuera de las empresas y universidades, proporcionando las ventajas asociadas al uso de las TIC's y dinamizando el acceso a la información y conocimiento.

De otra parte, las universidades corporativas muestran una tendencia de crecimiento que, se espera, puede llevarlas a equipararse en tamaño con las universidades tradicionales en el largo plazo. Estas tienen la ventaja de ofrecer soluciones específicas y aplicadas a los problemas propios de las organizaciones.

De manera general, los resultados obtenidos indican que hay amplias investigaciones alrededor de los entornos virtuales de aprendizaje, se sigue avanzando en las universidades corporativas y existe un campo abierto y creciente hacia los sistemas integrales de aprendizaje en las organizaciones. Aunque la revisión presentada aquí no es exhaustiva, se espera sirva como fundamentación teórica para desarrollos futuros en los temas relacionados.

\section{Referencias}

ABEL, A. L., and LI, J. (2012). « Exploring the Corporate University Phenomenon: Development and Implementation of a Comprehensive Survey ». Human Resource development Quarterly, 23(1), pp. 103128.

ACEYTUNO, M., y CACERES, R. (2012). « Los modelos europeos de transferencia de tecnología universidad-empresa ». Revista de Economía Mundial, pp. 215-238.

ALLEN, M. (2002). «What is a corporate university and why should an organization have one? ". The corporate university handbook, GloblalCCU, p. 9.

ANDRESEN, M. (2007). «Diversity learning, knowledge diversity and inclusion». Equal Opportunities International, pp. 743 - 760.

ANDRESEN, M., and LICHTENBERGER, B. (2007). "The corporate university landscape in Germany». Journal of Workplace Learning, 19(2), pp. 109-123.

ARGYRIS , C., and SCHON, D. (1996). Organizational learning II: Theory, method and practice. Addison Wesley.

ARGYRIS, C., and SCHON, D. (1978). Organizational Learning: A theory of action perspective. Addison Wesley.
BLASS, E. (2005). «The rise and rise of the corporate university». Journal of European Industrial Training, 29(1), pp. 58-74.

CAMPOS, H., PARELLADA, F., NUÑO, J., y PALMA, Y. (2011). «Empresas de base tecnológica derivadas de la universidad: el vínculo empresario - oportunidad tecnológica». Revista de Administração e Inovação, pp. 117-141.

CORREA, J., ARANGO, M., y ALVAREZ, K. (2012). «Metodología de valoración para proyectos de transferencia tecnológica universitaria.caso aplicado - Universidad de Antioquia». rev.fac.cienc.econ., pp. 91-106.

CYERT, R., and MARCH, J. G. (1992). A Behavioral Theory of the Firm (2 ed.). Malden,MA: Wiley-Blackwell.

DAVENPORT, T. H., and PRUSAK, L. (1998). Working Knowledge. Cambridge: Harvard Business Review Press.

DEALTRY , R. (2001). «Configuring the corporate university - managing a portfolio of thinking schools». Journal of workplace learning, 13(1), pp. $30-38$.

DEALTRY, R. (2000). «Case research into corporate university developments». Journal of Workplace Learning, 12(6), pp. 252-257.

DEALTRY, R. (2001). «How to configure the corporate university for success». Journal of Workplace Learning, 13(2), pp. 73-79.

DEALTRY, R. (2002). «Managing the corporate university learning curve». Journal of workpace learning, 14(2), pp. 76-81.

DEALTRY, R. (2002). «Managing the corporate university watershed». Journal of Workplace Learning, 14(6), pp. 256- 261.

DEALTRY, R. (2006). "The corporate university's role in managing an epoch in learning organisation innovation». Journal of Workplace Learning, 18(5), pp. 313-320.

DIXON, N. M. (1992). Organizational Leaming: A Review of the Literature with Implications for HRD Professionals. Human resource development, 3(1), pp. 29-41.

EASTERBY-SMITH, M., CROSSAN, M., and NICCOLINI, D. (2000). «Organizational Learning: Debates 
Past, Present And Future». Journal of Management Studies, 37(6), pp. 783-796.

EPPLE, D., ARGOTE, L., and DEVADAS , R. (1991). «Organizational learning curves: a method for investigating intra-plant transfer of knowledge acquired through learning by doing». Organization Science, 2(1), pp. 58-70.

FARIAS, G., y MONTOYA, J. (2009). «Gestión de un entorno virtual de aprendizaje para el desarrollo de competencias profesionales interculturales: una experiencia de educación superior entre México y España». Apertura, 1(1), pp. 6-19.

FERREIRO, V., BRITO, J., GARAMBULLO, A., y MARTINEZ, C. (2012). «Proyectos de vinculación escuela-empresa como estrategia de apoyo en la calidad del proceso enseñanza aprendizaje de la educación superior». Revista Internacional Administracion \& Finanzas, 5(3), pp.119-133.

GILBERT, D. (2013). « The Generation of Public Intellectuals: Corporate Universities, Graduate Employees and the Academic Labor Movement». Labor Studies Journal, 38(1), pp. 32-46.

GINANI, F., GADELHA, R., and AUGUSTO, G. (2012). « Use of Clinical Cases in a Virtual Learning Environment as an Approach to Teaching Human Embryology». Int. J. Morphol, 30(4), pp.1395-1398.

GUERCI, M., BARTEZZAGHI, E., and SOLARI, L. (2010). « Training evaluation in Italian corporate universities: a stakeholder-based analysis ». International Journal of Training and Development, 14(4), pp. 291-308.

GUTIERREZ, J., y BERRIO, O. (2011). « Punto de inflexión entre empresas y universidades ante la relación Universidad, Empresa y Estado en Colombia ». Univ. Empresa, 13(21), pp.167-191.

HAAS, C., y DE OLIVEIRA, R. (2008). « Universidade Corporativa: espaço diferenciado de aprendizagem no trabalho ». Pensamento \& Realidade, 11(3), pp. 95-11.

HOLLAND, P., and PYMAN, A. (2006). " Corporate universities: a catalyst for strategic human resource development? » Journal of European Industrial Training, 30(1), pp. 19-31.

HONG, J. (1999). « Structuring for organizational learning ». The learning organization, 6(4), pp. 173-185.
HUBER, G. P. (1991). « Organizational learning: the contributing processes and the literatures ». Organization Science, 2(1), pp. 88-115.

HURLEY, R. F., and M HULT, G. T. (1998). « Innovation, Market Orientation, and Organizational Learning: An integration and Empirical Examination ». Journal of marketing, 62(3), pp. 42-54.

IBERNÓN, F., SILVA, P., y GUZMÁN, C. (2011). « Competencias en los procesos de enseñanza, aprendizaje virtual y semipresencial ». Revista Científica de Educomunicación, 18(36), pp. 107-114.

JEREZ GOMEZ, P., CESPEDES LLORENTE, J., y VALLE CABRERA, R. (2003). " Organizational learning capability: a proposal of measurement ". Business Resarch, 58(1), pp. 715-725.

LAGE, M., y GODINHO, M. (2010). « Variations of the Kanban System: Literature Review and Classification ». International Journal of Production Economics, 125(1), pp. 13-21.

MACPHERSON, A., and HOMAN, G. (2005). « E-learning in the corporate university ». Journal of European Industrial Training, 29(1), pp. 75-90.

NIXON, J. C., and HELMS, M. M. (2002). « Corporate universities vs higher education institutions ". Industrial and commercial training, 34(4), pp. 144-150.

ORTEGA HURTADO, F., y LOCANO BOTERO, F. (2011). « Un modelo de control de gestion hacia el aprendizaje organizacional ». Dimensión empresarial, 9(1), pp. 17-27.

ÖRTENBLAD, A. (2001). « On differences between organizational learning and learning organization ». The learning organization, 8(3), pp. 125-133.

PHANG, C. W., KANKANHALLI, A., and ANG, C. (2008). " Investigating organizational learning in eGovernment projects: A multi-theoretic approach ». Strategic Information Systems, 17(2), pp. 99-123.

PRINCE, C., and STEWART, J. (2002). « Corporate universities - an analytical framework ». Journal of Management, 21(10), pp. 794-811.

RAZZAQ, K., ZAREEN, M., and RAMZAN, M. (2013). « Virtual learning: emerging trend in education sector of Pakistan ». Interdisciplinary Journal of Contemporary Research in Business, 4(10), pp. 856-875.

REINOSO, J. (2012). « Aplicación de un ambiente virtual de aprendizaje orientado a la formación empre- 
sarial ». Estudios Gerenciales, 28(122), pp.105-119.

RODRÍGUEZ, S. (2009). « Aviva España: Universidad Corporativa ». Capital humano, (238), pp. 36-41.

ROGERS, L. (2011). «Developing simulations in multi-user virtual environments to enhance healthcare education ». British Journal of Educational Technology, 42(4), pp. 608-615.

RYAN, L. (2007). «Developing a qualitative understanding of university corporate education ". Management Decision, 45(2), pp. 153 - 160.

RYAN, L. (2009). «Exploring the growing phenomenon of university-corporate education partnerships». Management Decision, 47(8), pp. 1313-1322.

SANDELANDS, E. (1997). «Developing a Robust Model of the Virtual Corporate University ». Journal of Knowledge Management, 1(3), pp. 181-188.

SHAM, C. (2007). «An exploratory study of corporate universities in China ». Journal of Workplace Learning, 19(4), pp. 257 - 264.
SHAW, S. (2005). « The corporate university ». Journal of European Industrial Training, 29(1), pp. 21 -39.

STUMPF, S. A. (1998). "Corporate universities of the future ». Career Developmente International, 3(5), pp. 206-211.

VÁSQUEZ, C., y ARANGO, S. (2012). «Estrategias de participación e interacción en entornos virtuales de aprendizaje ». ANAGRAMAS, 10(20), pp. 95-108.

WALTON, J. (2005). « Would the real corporate university please stand up? " Journal of European Industrial Training, 29(1), pp. 7 - 20.

WELLER, M. (2007). Virtual learning environments: using, choosing and developing your VLE. Londres: Routledge.

XUEJUN, J. (2009). "Corporate universities in China: processes, issues and challenges ». Journal of Workplace Learning, 21(2), pp. 166 - 174. 